PROCEEDINGS OF THE

AMERICAN MATHEMATICAL SOCIETY

Volume 135, Number 8, August 2007, Pages 2317-2326

S 0002-9939(07)08735-7

Article electronically published on March 21, 2007

\title{
ON A CONJECTURE OF KOIKE ON IDENTITIES BETWEEN THOMPSON SERIES AND ROGERS-RAMANUJAN FUNCTIONS
}

\author{
KATHRIN BRINGMANN AND HOLLY SWISHER
}

(Communicated by Ken Ono)

\begin{abstract}
One of the many amazing things Ramanujan did in his lifetime was to list 40 identities involving what are now called the Rogers-Ramanujan functions $G(q)$ and $H(q)$ on one side, and products of functions of the form $Q_{m}=\prod_{n=1}^{\infty}\left(1-q^{m n}\right)$ on the other side. The identities are rather complicated and seem too difficult to guess. Recently however, Koike devised a strategy for finding (but not proving) these types of identities by connecting them to Thompson series. He was able to conjecture many new Rogers-Ramanujan type identities between $G(q)$ and $H(q)$, and Thompson series. Here we prove these identities.
\end{abstract}

\section{INTRODUCTION AND STATEMENT OF RESULTS}

One of the many amazing things Ramanujan did in his lifetime was to list 40 identities involving the Rogers-Ramanujan functions

$$
\begin{aligned}
G(q) & :=\prod_{n=0}^{\infty} \frac{1}{\left(1-q^{5 n+1}\right)\left(1-q^{5 n+4}\right)}, \\
H(q) & :=\prod_{n=0}^{\infty} \frac{1}{\left(1-q^{5 n+2}\right)\left(1-q^{5 n+3}\right)} .
\end{aligned}
$$

One example of such an identity is the following one:

$$
G(q) G\left(q^{4}\right)+q H(q) H\left(q^{4}\right)=q^{1 / 12}\left(\frac{\eta(2 z)^{2}}{\eta(z) \eta(4 z)}\right)^{2} \quad\left(q=e^{2 \pi i z}\right),
$$

where $\eta(z):=q^{1 / 24} \prod_{n=1}^{\infty}\left(1-q^{n}\right)$ is Dedekind's $\eta$-function. Many people have contributed to proving Ramanujan's identities (for example, Bia89, Dar21, Rog21, and Wat33]).

According to Birch [Bir75] these identities seem too complicated to guess even for one with Ramanujan's incredible instinct for formulae. However, Koike recently made an interesting observation which enabled him to conjecture several new identities of the same type as Ramanujan's. Koike observed that certain $\eta$ products which are connected to Thompson series in Conway and Norton's famous paper "Monstrous Moonshine" CN79 appear on both sides of these identities. His method yields some of the identities in Ramanujan's list as well as new ones (see Koi04]). Here we prove the new identities conjectured by Koike.

Received by the editors January 31, 2006 and, in revised form, March 27, 2006.

2000 Mathematics Subject Classification. Primary 11F22, 33D15, 11 F03. 
Let $M$ denote the monster, the largest sporadic finite simple group. For every $g \in M$, the Thompson series $T_{g}$ is defined by

$$
T_{g}:=q^{-1}+0+H_{1}(g) q+H_{2}(g) q^{2}+\cdots,
$$

where the $H_{i}(g)$ are character values for certain representations $H_{i}$ of $M$ (see CN79).

Let $N$ be a positive integer, and let $e$ be a Hall divisor of $N$ (written $e \| N$ ), which is a positive divisor of $N$ such that $(e, N / e)=1$. An Atkin-Lehner involution $W_{e}$ is a matrix of the form

$$
W_{e}:=\left(\begin{array}{cc}
a e & b \\
c N & d e
\end{array}\right)
$$

such that $\operatorname{det} W_{e}=e$.

Consider subgroups of $\mathrm{PSL}_{2}(\mathbb{R})$ obtained by extending $\Gamma_{0}(N)$ by certain AtkinLehner involutions. In particular, if $S$ is a subset of Hall divisors of $N$, we write $N+S$ to denote the group generated by $\Gamma_{0}(N)$ and all Atkin-Lehner involutions $W_{e}$ for $e \in S$. For shorthand, we write $N+$ when we adjoin all Atkin-Lehner involutions.

For $N+S \notin\{25-, 49+49,50+50\}$, let $X_{N+S}$ be the Riemann surface obtained by compactifying $\mathcal{H} /(N+S)$. If $X_{N+S}$ has genus zero, then by the work of Borcherds, there is an element $g \in M$ such that $T_{g}$ is the normalized generator of the function field of $X_{N+S}$. In this case we define

$$
T_{N+S}:=T_{g} .
$$

Let

$$
\begin{aligned}
g(z) & :=q^{-1 / 60} G(q), \\
h(z) & :=q^{11 / 60} H(q) .
\end{aligned}
$$

Moreover, if $r \equiv \pm s(\bmod 5)$ we define the functions $u_{r, s}(z)$ by

$$
u_{r, s}(z):=\left\{\begin{array}{lll}
g(r z) g(s z)+h(r z) h(s z) & \text { for } r+s \equiv 0 & (\bmod 5), \\
g(r z) h(s z)-h(r z) g(s z) & \text { for } r-s \equiv 0 & (\bmod 5) .
\end{array}\right.
$$

The purpose of this paper is to prove the following conjecture made by Koike (see [Koi04]), which we now state as a theorem.

Theorem 1.1. Let $M$ be the monster group, and suppose $N$ is among the following set of orders of elements of $M$ :

$$
\left\{\begin{array}{l}
4,6,9,14,19,21,24,26,29,31,34,36,39 \\
41,44,46,51,54,56,59,66,69,71,119
\end{array}\right\} .
$$

Then there exists an element $g \in M$ of order $N$ and a constant $c_{N}$ depending on $N$ such that $T_{g}+c_{N}$ can be expressed as a product of Rogers-Ramanujan functions $u_{r, s}(z)$. (See Appendix $\mathrm{A}$ for a complete list of the identities and the constants $c_{N}$.) A.

As an example of Theorem 1.1 we give the first of Koike's identities in Appendix

$$
\left(u_{4,1}(z)\right)^{12}=T_{4+}+24=\frac{\eta(2 z)^{48}}{\eta(z)^{24} \eta(4 z)^{24}},
$$

where the second equality comes from Conway and Norton's Monstrous Moonshine CN79. 
Remark. The identities (A.1), A.2), A.4 , A.13, (A.15), (A.16), and A.21 in Appendix A already follow from the Ramanujan identities [Bir75].

\section{Preliminaries}

Before we give the proof of Theorem 1.1 we require some facts about modular forms. See BBP87, Bia89, Mar96] for more details.

If $f$ is a modular form on a congruence subgroup $\Gamma$ and $\zeta$ is a cusp of $\Gamma$, then $f$ has a Fourier expansion at $\zeta$ of the form

$$
\sum_{m \geq m_{0}} c(m) q_{h}^{m}
$$

where $q_{h}:=e^{2 \pi i z / h}, m_{0} \in \mathbb{Z}, c\left(m_{0}\right) \neq 0$, and $h:=h(\Gamma, \zeta)$ is the width of $\Gamma$ at the cusp $\zeta$. We let $\operatorname{Ord}_{\Gamma}(f, \zeta):=m_{0}$ and call it the relative order of vanishing of $f$ with respect to $\Gamma$. Furthermore, we define ord $(f, \zeta)$, the invariant order of $f$ at $\zeta$, to be the smallest power of $q:=e^{2 \pi i z}$ appearing in the Fourier expansion of $f$ at $\zeta$. Thus the two orders satisfy

$$
\operatorname{Ord}_{\Gamma}(f, \zeta)=h(\Gamma, \zeta) \cdot \operatorname{ord}(f, \zeta)
$$

When we consider $z \in \mathcal{H}$, the upper half-plane, $\operatorname{ord}(f, z)$ is the usual order of $f$ as an analytic function at $z$. If $a$ is the order of the stabilizer of $z$ in $\Gamma$ (considered as a subgroup of $\left.\operatorname{PSL}_{2}(\mathbb{Z})\right)$ and $f$ is a modular form on $\Gamma$, then for every $z \in \mathcal{H}$ we define [BBP87]

$$
\operatorname{Ord}_{\Gamma}(f(z), z):=\frac{\operatorname{ord}(f, z)}{a} \geq 0 .
$$

A meromorphic modular form on $\Gamma_{0}(N)$ is called weakly holomorphic if its poles, if there are any, are supported at the cusps of $\Gamma_{0}(N)$. A complete set of representatives for the cusps of $\Gamma_{0}(N)$ is given by (see Mar96])

$$
\left\{\frac{a_{c}}{c}: c \mid N, 1 \leq a_{c} \leq N, \operatorname{gcd}\left(a_{c}, N\right)=1, a_{c} \text { distinct }(\bmod \operatorname{gcd}(c, N / c))\right\} .
$$

We now state the modularity of the functions $u_{r, s}$. For this we define the multiplier $\chi_{r, s}$ on matrices in $\Gamma_{0}([r, s])$ :

$$
\chi_{r, s}\left(\left(\begin{array}{ll}
\alpha & \beta \\
\gamma & \delta
\end{array}\right)\right):= \begin{cases}\exp \left(\frac{7 \pi i(r+s)}{6}(\beta \delta+(\gamma / r s)(\alpha+\delta-\beta \gamma \delta-3))\right) & \text { if } \gamma \text { odd } \\
\exp \left(\frac{7 \pi i(r+s)}{6}\left(\beta \delta+(\gamma / r s)\left(\alpha-\delta-\alpha \delta^{2}\right)\right)\right) & \text { if } \delta \text { odd }\end{cases}
$$

The following two results are due to Biagioli (Theorems 2.4 and 3.3 resp. of Bia89).

Lemma 2.1. If $r, s$ are positive integers, and $[r, s]$ denotes the least common multiple of $r$ and $s$, then the functions $u_{r, s}(z)$ are weakly holomorphic modular functions on $\Gamma_{0}([r, s])$ with multiplier system $\chi_{r, s}$.

Theorem 2.2. If $f(z)$ is one of either $g(z)$ or $h(z)$ defined in (1.4) and (1.5), and $a, c$ are integers with $(a, c)=1$, then

$$
\operatorname{ord}\left(f(z), \frac{a}{c}\right) \in\left\{\frac{11}{16},-\frac{1}{60}\right\} \text {. }
$$


For our purposes we don't need the exact criteria for when each case occurs. Combining this theorem with the fact that for all modular forms $f(z)$ of weight 0 , and all $m \in \mathbb{N}$ we have

$$
\operatorname{ord}\left(f(m z), \frac{a}{c}\right)=\frac{\operatorname{gcd}(m, c)^{2}}{m} \cdot \operatorname{ord}\left(f(z), \frac{m a}{c}\right)
$$

gives the following bounds for the invariant order of vanishing of the $u_{r, s}(z)$ :

$$
\begin{gathered}
\operatorname{ord}\left(u_{r, s}(z), \frac{a}{c}\right) \geq-\frac{1}{60}\left(\frac{\operatorname{gcd}(r, c)^{2}}{r}+\frac{\operatorname{gcd}(s, c)^{2}}{s}\right), \\
\operatorname{ord}\left(u_{r, s}(z)^{-1}, \frac{a}{c}\right) \geq-\frac{11}{16}\left(\frac{\operatorname{gcd}(r, c)^{2}}{r}+\frac{\operatorname{gcd}(s, c)^{2}}{s}\right) .
\end{gathered}
$$

For the group $\Gamma_{0}(N)$ we have the formula [Mar96] for the width of $\Gamma_{0}(N)$ at the cusp $\frac{a}{c}$,

$$
h_{\frac{a}{c}}:=h\left(\Gamma_{0}(N), \frac{a}{c}\right)=\frac{N}{\operatorname{gcd}\left(c^{2}, N\right)} .
$$

The largest possible width at $\frac{a}{c}$ is thus $\frac{N}{c}$, and from this we easily obtain the following proposition.

Proposition 2.3. Let $N=[r, s]$ and let $\frac{a}{c}$ be a cusp of $\Gamma_{0}(N)$. Then

$$
\operatorname{Ord}_{\Gamma_{0}(N)}\left(u_{r, s}(z)^{n}, \frac{a}{c}\right) \geq \begin{cases}-\frac{n N}{30} & \text { if } n \geq 1, \\ \frac{n N \cdot 11}{8} & \text { if } n \leq-1 .\end{cases}
$$

In addition, much is known about the modularity of $\eta$-quotients (see Mar96, Ono04). The following theorem (see, e.g., Ono04, page 18) describes when an $\eta$-quotient is modular.

Theorem 2.4. Assume that $f(z)=\prod_{\delta \mid N} \eta(\delta z)^{r_{\delta}}\left(N \in \mathbb{N}, r_{\delta} \in \mathbb{Z}\right)$ is an $\eta$-quotient such that $k:=\frac{1}{2} \sum_{\delta \mid N} r_{\delta} \in \mathbb{Z}$,

$$
\sum_{\delta \mid N} \delta r_{\delta} \equiv 0 \quad(\bmod 24)
$$

and

$$
\sum_{\delta \mid N} \frac{N}{\delta} r_{\delta} \equiv 0 \quad(\bmod 24)
$$

Then $f(z)$ is a meromorphic modular form of weight $k$ for the group $\Gamma_{0}(N)$ with Nebentypus character $\chi$ given by

$$
\chi(d):=\left(\frac{(-1)^{k} s}{d}\right)
$$

for $s:=\prod_{\delta \mid N} \delta^{r_{\delta}}$.

We can also calculate the orders of vanishing of $\eta$-quotients at cusps of $\Gamma_{0}(N)$ by the following theorem.

Theorem 2.5. If $f(z)$ is an $\eta$-quotient that satisfies the conditions of Theorem 2.4, then

$$
\operatorname{Ord}_{\Gamma_{0}(N)}\left(f, \frac{a}{c}\right)=\frac{N}{24 \operatorname{gcd}\left(c^{2}, N\right)} \sum_{\delta \mid N} \frac{\operatorname{gcd}(c, \delta)^{2} \cdot r_{\delta}}{\delta} .
$$




\section{Proof of Theorem 1.1}

3.1. Overview of method. To prove identities of the type

$$
\prod u_{r, s}=T_{N+S}
$$

for a given $N$, we consider $g_{N}(z)=\prod u_{r, s}-T_{N+S}$ and prove that $g_{N}(z)=0$. To do this, we realize $g_{N}(z)$ as a weight 0 modular form on $\Gamma_{0}(N)$ and then contradict the valence formula for $g_{N}(z)$ (see [Bia89] and equation (3.1)).

The Thompson series $T_{N+S}$ can be viewed as a weakly holomorphic modular function with respect to the group $N+S$ (see Kim04). Since this group contains $\Gamma_{0}(N)$ we may view $T_{N+S}$ as a meromorphic modular function on $\Gamma_{0}(N)$.

Similarly, Lemma 2.1 implies that each quotient of $u_{r, s}(z)$ functions in Koike's identities is a meromorphic modular function on $\Gamma_{0}(N)$ for some $N$ with multiplier $\chi$, where $\chi$ is the corresponding quotient of multipliers from equation (2.4). A direct calculation in each case shows that in fact the multiplier system is trivial for each quotient. Thus for each $N$ if $g_{N}(z)$ is defined to be the difference of the left-hand side and the right-hand side of the corresponding identity from Appendix A. then $g_{N}(z)$ is a meromorphic modular function on $\Gamma_{0}(N)$. Thus the valence formula gives that if $g_{N}(z)$ is not zero, then

$$
\sum_{\tau \in \mathcal{H}} \operatorname{Ord}_{\Gamma_{0}(N)}\left(g_{N}(z), \tau\right)+\sum_{\frac{a}{c} \nsim \infty} \operatorname{Ord}_{\Gamma_{0}(N)}\left(g_{N}(z), \frac{a}{c}\right)+\operatorname{Ord}_{\Gamma_{0}(N)}\left(g_{N}(z), \infty\right)=0,
$$

where the second sum runs over a set of inequivalent cusps $\frac{a}{c}$ of $\Gamma_{0}(N)$ that are not equivalent to $\infty$.

Since $g_{N}(z)$ is holomorphic on $\mathcal{H}$, the first summand is clearly positive. Our goal is to obtain lower bounds for $\operatorname{Ord}_{\Gamma_{0}(N)}\left(g_{N}(z), \frac{a}{c}\right)$ for cups $\frac{a}{c} \not \infty$. Then we can contradict (3.1) by showing that $\operatorname{Ord}_{\Gamma_{0}(N)}\left(g_{N}(z), \infty\right)$ is "big enough" by computing coefficients of $g_{N}(z)$ at infinity. In the following sections we will be more precise about the number of coefficients required in each case.

To get a lower bound for $\operatorname{Ord}_{\Gamma_{0}(N)}\left(g_{N}(z), \frac{a}{c}\right)$, we simply take the minimum of the lower bounds for the quotient of $u_{r, s}$ functions and the Thompson series on either side of the corresponding identity.

By Proposition 2.3 we easily obtain lower bounds for the relative orders of vanishing of the $u_{r, s}$ quotients that appear in the left-hand sides of the identities. For the Thompson series, we use one of the following methods.

Remark. We first note that from the Ramanujan identities one can prove the identities for $N=4,6,14,39,44,46,66$. For example it is not hard to see that (1.3) is equivalent to the identity for $N=4$.

3.2. Group $\mathbf{N}+$ with $\mathbf{N}$ square-free. Here we consider the identities for squarefree $N$, where the Thompson series $T_{N+}$ is associated to the group $N+$ with all Atkin-Lehner involutions adjoined. These are the identities for

$$
N \in\{19,21,26,29,31,34,41,46,51,59,69,71,119\} .
$$

When $N$ is square-free, (2.3) gives that the set of cusps for $\Gamma_{0}(N)$ is given by

$$
\left\{\frac{1}{m}: m \mid N\right\} \text {. }
$$


Proposition 3.1. For $N \in\{19,21,26,29,31,34,41,46,51,59,69,71,119\}$ we have

$$
\operatorname{Ord}_{\Gamma_{0}(N)}\left(T_{N+}(z), \frac{1}{m}\right)=-1 .
$$

Proof. First we recall that if $M:=\left(\begin{array}{ll}a & b \\ c & d\end{array}\right) \in \mathrm{SL}_{2}(\mathbb{R})$ and $z \in \mathcal{H}$, then

$$
M z:=\frac{a z+b}{c z+d} .
$$

If we let

$$
A_{m}:=\left(\begin{array}{cc}
1 & 0 \\
m & 1
\end{array}\right) \in \mathrm{SL}_{2}(\mathbb{Z})
$$

then the Fourier expansion of $g_{N}\left(A_{m} z\right)$ at $\infty$ reflects the behavior of $g_{N}(z)$ at the cusp $1 / m$. Since $N$ is square-free, we can find for $e:=N / m$ integers $x, y$ such that $x e-y m=1$. Thus the matrix

$$
W_{e}:=\left(\begin{array}{cc}
x e & -y \\
-N & e
\end{array}\right)
$$

is an Atkin-Lehner involution with determinant $e$, and so $T_{N+}$ is invariant under $W_{e} \in N+$. Since $W_{e} A_{m}=\left(\begin{array}{ll}1 & y \\ 0 & e\end{array}\right)$, we have

$$
T_{N+}\left(A_{m} z\right)=T_{N+}\left(\left(\begin{array}{ll}
1 & y \\
0 & e
\end{array}\right) z\right)
$$

Recalling that ord $\left(T_{N+}, \infty\right)=-1$, this yields that ord $\left(T_{N+}, \frac{1}{m}\right)=-1 / e$. Multiplying by the width $e$ from equation (2.6) gives the desired result.

3.3. Remaining cases. The 5 cases not yet covered are $N=9,24,36,54,56$. For these we use the fact that we can write the Thompson series as a linear combination of $\eta$-quotients (see [CN79]). Calculating via Theorem [2.5, we obtain the orders of vanishing we need. The case where $N=54$ must be treated more delicately and is considered in Section 3.5

Let $N \neq 4,6,14,39,44,46,66$ (the cases already proved with the Ramanujan identities). After computing the necessary bounds for the orders of vanishing for each Thompson series (or associated $\eta$-quotient) and comparing them with the bounds from Proposition 2.3 for the $u_{r, s}(z)$ quotients, we obtain the following table giving the number of coefficients of the Fourier expansion of $g_{N}(z)$ at $\infty$ that we need to compute in order to prove that $g_{N}(z)=0$. The resulting computations are done easily using Maple.

\begin{tabular}{cccccccccccccccccc}
$N$ & 9 & 19 & 21 & 24 & 26 & 29 & 31 & 34 & 36 & 41 & 51 & 54 & 56 & 59 & 69 & 71 & 119 \\
\hline coefficients & 6 & 2 & 13 & 17 & 11 & 2 & 4 & 14 & 558 & 3 & 11 & 8 & 8 & 2 & 14 & 3 & 24
\end{tabular}

Remark. The reason that we compute so many coefficients for $N=36$ is due to a combination of the fact that 36 has many divisors, and because the identity in this case has a $u_{r, s}(z)$ function in the denominator. Proposition 2.3 gives poor bounds for $u_{r, s}(z)$ functions in the denominator. We could get better bounds, but the resulting notation and complication is unnecessary for the proof. 
3.4. Example. Here we demonstrate the argument for the case $N=9$. Using Lemma 2.1 we see that $\left(u_{9,1}(z)\right)^{6}$ is a modular function on $\Gamma_{0}(9)$ with trivial multiplier. Also, from Theorem 2.4 we see that the functions $\frac{\eta(z)^{3}}{\eta(9 z)^{3}}$ and $\frac{\eta(9 z)^{3}}{\eta(z)^{3}}$ are modular functions on $\Gamma_{0}(9)$ with trivial multiplier, yielding that

$$
f_{9}(z):=\frac{\eta(z)^{3}}{\eta(9 z)^{3}}+27 \frac{\eta(9 z)^{3}}{\eta(z)^{3}}+9
$$

is also. Thus we have that $g_{9}(z):=\left(u_{9,1}(z)\right)^{6}-f_{9}(z)$ is a modular function on $\Gamma_{0}(9)$.

The valence formula (3.1) gives that if $g_{9}(z) \neq 0$, then

$$
\sum_{\tau \in \mathcal{H}} \operatorname{Ord}_{\Gamma_{0}(9)}\left(g_{9}(z), \tau\right)+\sum_{\frac{a}{c} \nsim \infty} \operatorname{Ord}_{\Gamma_{0}(9)}\left(g_{9}(z), \frac{a}{c}\right)+\operatorname{Ord}_{\Gamma_{0}(9)}\left(g_{9}(z), \infty\right)=0 .
$$

The first summand is clearly nonnegative since $g_{9}(z)$ is holomorphic on $\mathcal{H}$. We now estimate the second summand. By (2.3), we see that a set of representatives of the cusps of $\Gamma_{0}(9)$ is

$$
\left\{1, \frac{1}{3}, \frac{2}{3}, \frac{1}{9}\right\}
$$

where the cusp $1 / 9$ is equivalent to $\infty$. Using Proposition 2.3 we see that for any cusp $a / c$ of $\Gamma_{0}(9)$,

$$
\operatorname{Ord}_{\Gamma_{0}(9)}\left(\left(u_{9,1}(z)\right)^{6}, \frac{a}{c}\right) \geq-\frac{9}{5} .
$$

Then we observe from a repeated application of Theorem 2.5 that $\operatorname{Ord}_{\Gamma_{0}(9)}\left(f_{9}(z), \frac{a}{c}\right) \geq-1$, and so we have the bound

$$
\operatorname{Ord}_{\Gamma_{0}(9)}\left(g_{9}(z), \frac{a}{c}\right) \geq-\frac{9}{5} .
$$

Since there are 3 cusps of $\Gamma_{0}(9)$ which are not equivalent to $\infty$, the second term is larger than -6 .

One can easily compute Fourier coefficients of $g_{9}$ using Maple to show that $\operatorname{Ord}_{\Gamma_{0}(9)}\left(g_{9}(z), \infty\right)>6$, and thus the valence formula (3.1) is contradicted. This gives that $g_{9}(z)=0$, which proves (A.3).

3.5. The case $N=54$. In the case $N=54$, we argue a little differently. Using (2.3), we easily see that a complete set of representatives of cusps of $\Gamma_{0}(54)$ is given by

$$
\left\{1, \frac{1}{2}, \frac{1}{3}, \frac{5}{3}, \frac{1}{6}, \frac{5}{6}, \frac{1}{9}, \frac{5}{9}, \frac{1}{18}, \frac{5}{18}, \frac{1}{27}, \frac{1}{54}\right\} .
$$

As in Section 3.4, we see that $u_{54,1}(z) u_{27,2}(z)$ is a modular function on $\Gamma_{0}(54)$ whose order of vanishing is $-\frac{7}{5}$ at the cusps

$$
1, \frac{1}{2}, \frac{1}{27}, \frac{1}{54},
$$

and is $-\frac{1}{5}$ at the cusps

$$
\frac{1}{3}, \frac{5}{3}, \frac{1}{6}, \frac{5}{6}, \frac{1}{9}, \frac{5}{9}, \frac{1}{18}, \frac{5}{18} .
$$

Thus we are left to compute the order of vanishing of

$$
f_{54}(z):=\frac{\eta(z+1 / 3) \cdot \eta(2 z+1 / 3) \cdot \eta(3 z+2 / 3) \cdot \eta(6 z+2 / 3)}{\eta(3 z) \cdot \eta(6 z) \cdot \eta(9 z) \cdot \eta(18 z)}
$$


at all cusps of $\Gamma_{0}(54)$. At the cusps $1, \frac{1}{2}, \frac{1}{27}, \frac{1}{54}$, we argue as in Section 3.2. Since the other cusps are all treated in the same manner we will only give the proof for the cusp $\frac{1}{3}$. Clearly the matrix $M:=\left(\begin{array}{ll}1 & 0 \\ 3 & 1\end{array}\right)$ maps $\infty$ to $\frac{1}{3}$. We now consider

$$
f_{54}(z)^{24}=\frac{\Delta(z+1 / 3) \cdot \Delta(2 z+1 / 3) \cdot \Delta(3 z+2 / 3) \cdot \Delta(6 z+2 / 3)}{\Delta(3 z) \cdot \Delta(6 z) \cdot \Delta(9 z) \cdot \Delta(18 z)}
$$

where $\Delta(z):=\eta^{24}(z)$ is the usual $\Delta$-function. It is easy to see that

$$
f_{54}(M z)^{24}=\frac{\left.\left.\left.\left.\Delta\right|_{12}\left(\begin{array}{ll}
3 & 1 \\
0 & 3
\end{array}\right) M z \cdot \Delta\right|_{12}\left(\begin{array}{ll}
6 & 1 \\
0 & 3
\end{array}\right) M z \cdot \Delta\right|_{12}\left(\begin{array}{ll}
9 & 2 \\
0 & 3
\end{array}\right) M z \cdot \Delta\right|_{12}\left(\begin{array}{cc}
18 & 2 \\
0 & 3
\end{array}\right) M z}{\left.\left.\left.\left.3^{24} \Delta\right|_{12}\left(\begin{array}{cc}
3 & 0 \\
0 & 1
\end{array}\right) M z \cdot \Delta\right|_{12}\left(\begin{array}{ll}
6 & 0 \\
0 & 1
\end{array}\right) M z \cdot \Delta\right|_{12}\left(\begin{array}{ll}
9 & 0 \\
0 & 1
\end{array}\right) M z \cdot \Delta\right|_{12}\left(\begin{array}{cc}
18 & 0 \\
0 & 1
\end{array}\right) M z},
$$

where for a function $f: \mathcal{H} \rightarrow \mathbb{C}$, and a matrix $A:=\left(\begin{array}{ll}a & b \\ c & d\end{array}\right) \in \mathrm{SL}_{2}(\mathbb{R})$ we define the weight 12 slash operator $\left.\right|_{12}$ by

$$
\left.f\right|_{12} A z:=(a d-b c)^{6} \cdot(c z+d)^{-12} \cdot f(A z)
$$

Now we can compute the order of vanishing of $f_{54}(M z)^{24}$ at $\infty$ by using the transformation law for the $\Delta$-function. For example we have

$$
\begin{aligned}
\left.\Delta\right|_{12}\left(\begin{array}{ll}
3 & 1 \\
0 & 3
\end{array}\right) M z & =\left.\Delta\right|_{12}\left(\begin{array}{ll}
6 & 1 \\
9 & 3
\end{array}\right) z \\
& =\left.\Delta\right|_{12}\left(\begin{array}{ll}
-1 & 1 \\
-3 & 2
\end{array}\right)\left(\begin{array}{ll}
6 & 1 \\
9 & 3
\end{array}\right) z=\left.\Delta\right|_{12}\left(\begin{array}{ll}
3 & 2 \\
0 & 3
\end{array}\right) z=\zeta_{1} \cdot q+\ldots
\end{aligned}
$$

where $\zeta_{1}$ is some root of unity. In the same way we treat the other $\Delta$-factors. From this we can easily conclude that

$$
f_{54}(M z)=\zeta_{2}+O(q)
$$

where $\zeta_{2}$ is some root of unity. Thus we directly obtain that the order of vanishing of $f_{54}$ at $\frac{1}{3}$ is 0 . Treating the other cusps in the same way and comparing with our bounds for $u_{54,1}(z) u_{27,2}(z)$ gives us the bound -8 for $g_{54}(z)$ as in the Appendix. 


\section{Appendix A.}
(A.1) $\quad\left(u_{4,1}(z)\right)^{12}=T_{4+}+24=\frac{\eta(2 z)^{48}}{\eta(z)^{24} \eta(4 z)^{24}}$
$N=4$
(A.2) $\quad\left(u_{3,2}(z)\right)^{12}=T_{6+6}+12=\frac{\eta(2 z)^{12} \eta(3 z)^{12}}{\eta(z)^{12} \eta(6 z)^{12}}$
$N=6$
(A.3) $\quad\left(u_{9,1}(z)\right)^{6}=T_{9+}+6=\frac{\eta(z)^{3}}{\eta(9 z)^{3}}+27 \frac{\eta(9 z)^{3}}{\eta(z)^{3}}+9$
$N=9$
(A.4) $\quad\left(u_{14,1}(z)\right)^{4}=T_{14+14}+4=\frac{\eta(2 z)^{4} \eta(7 z)^{4}}{\eta(z)^{4} \eta(14 z)^{4}}$
$N=14$
(A.5) $\quad\left(u_{19,1}(z)\right)^{3}=T_{19+}+3$
$N=19$
(A.6) $\quad\left(u_{7,3}(z)\right)^{6}=T_{21+}=\frac{\eta(3 z)^{2} \eta(7 z)^{2}}{\eta(z)^{2} \eta(21 z)^{2}}+\frac{\eta(z)^{2} \eta(21 z)^{2}}{\eta(3 z)^{2} \eta(7 z)^{2}}-2$
$N=21$
(A.7) $\quad\left(u_{24,1}(z)\right)^{2} \cdot u_{6,4}(z)=T_{24+24}+2=\frac{\eta(2 z) \eta(3 z)^{2} \eta(8 z)^{2} \eta(12 z)}{\eta(z)^{2} \eta(4 z) \eta(6 z) \eta(24 z)^{2}}$
$N=24$
(A.8) $\quad\left(u_{13,2}(z)\right)^{4}=T_{26+}=\frac{\eta(2 z)^{2} \eta(13 z)^{2}}{\eta(z)^{2} \eta(26 z)^{2}}+\frac{\eta(z)^{2} \eta(26 z)^{2}}{\eta(2 z)^{2} \eta(13 z)^{2}}-2$
$N=26$
(A.9) $\quad\left(u_{29,1}(z)\right)^{2}=T_{29+}+2$
$N=29$
(A.10) $\left(u_{31,1}(z)\right)^{3}=T_{31+}$
$N=31$
(A.11) $\left(u_{34,1}(z) u_{17,2}(z)\right)^{2}=T_{34+}+2$
$N=34$
(A.12) $\frac{u_{36,1}(z)}{u_{9,4}(z)}=T_{36+36}+1=\frac{\eta(4 z) \eta(9 z)}{\eta(z) \eta(36 z)}$
$N=36$
(A.13) $\frac{u_{39,1}(z)}{u_{13,3}(z)}=T_{39+39}+1=\frac{\eta(3 z) \eta(13 z)}{\eta(z) \eta(39 z)}$
$N=39$
(A.14) $\left(u_{41,1}(z)\right)^{2}=T_{41+}$
$N=41$
(A.15) $u_{44,1}(z) u_{11,4}(z)=T_{44+}+1=\frac{\eta(2 z)^{4} \eta(22 z)^{4}}{\eta(z)^{2} \eta(4 z)^{2} \eta(11 z)^{2} \eta(44 z)^{2}}-1$
$N=44$
(A.16) $u_{46,1}(z) u_{23,2}(z)=T_{46+}=\frac{\eta(z) \eta(23 z)}{\eta(2 z) \eta(46 z)}+2 \frac{\eta(2 z) \eta(46 z)}{\eta(z) \eta(23 z)}+1$
$N=46$
(A.17) $u_{51,1}(z) u_{17,3}(z)=T_{51+}$
$N=51$
(A.18) $u_{54,1}(z) u_{27,2}(z)=T_{54+}+1=\frac{\eta(z+1 / 3) \eta(2 z+1 / 3) \eta(3 z+2 / 3) \eta(6 z+2 / 3)}{\eta(3 z) \eta(6 z) \eta(9 z) \eta(18 z)}$
$N=54$
(A.19) $u_{56,1}(z) u_{8,7}(z)=T_{56+}=\frac{\eta(2 z) \eta(4 z) \eta(14 z) \eta(28 z)}{\eta(z) \eta(7 z) \eta(8 z) \eta(56 z)}-1$
$N=56$
(A.20) $u_{59,1}(z)=T_{59+}+1$
$N=59$
(A.21) $\frac{u_{22,3}(z) u_{33,2}(z)}{u_{11,6}(z) u_{66,1}(z)}=T_{66+6,11,66}+1=\frac{\eta(2 z) \eta(3 z) \eta(22 z) \eta(33 z)}{\eta(z) \eta(6 z) \eta(11 z) \eta(66 z)}$
$N=66$
(A.22) $u_{69,1}(z) u_{23,3}(z)=T_{69+}+1$
$N=69$
(A.23) $u_{71,1}(z)=T_{71+}$
$N=71$
(A.24) $u_{119,1}(z) u_{17,7}(z)=T_{119+}+1$
$N=119$ 


\section{ACKNOWLEDGMENTS}

The authors would like to thank Ken Ono and Jeremy Rouse for helpful conversations and comments on earlier versions of this paper.

\section{REFERENCES}

[BBP87] Bruce C. Berndt, Anthony J. Biagioli, and James M. Purtilo, Ramanujan's modular equations of "large" prime degree, J. Indian Math. Soc. (N.S.) 51 (1987), 75-110 (1988). MR.MR988310 (90b:11038)

[Bia89] Anthony J. F. Biagioli, A proof of some identities of Ramanujan using modular forms, Glasgow Math. J. 31 (1989), no. 3, 271-295. MRMR1021804 (90m:11060)

[Bir75] B. J. Birch, A look back at Ramanujan's notebooks, Math. Proc. Cambridge Philos. Soc. 78 (1975), 73-79. MRMR0379372 (52 \#277)

[CN79] J. H. Conway and S. P. Norton, Monstrous moonshine, Bull. London Math. Soc. 11 (1979), no. 3, 308-339. MRMR554399 (81j:20028)

[CY96] Imin Chen and Noriko Yui, Singular values of Thompson series, Groups, difference sets, and the Monster (Columbus, OH, 1993), Ohio State Univ. Math. Res. Inst. Publ., vol. 4, de Gruyter, Berlin, 1996, pp. 255-326. MRMR1400423 (98a:11051)

[Dar21] H. B. C. Darling, Proof of certain identities and congruences enunciated by S. Ramanujan, Proc. London Math. Soc. (2) 19 (1921), 350-372.

[Kim04] Chang Heon Kim, Borcherds products associated with certain Thompson series, Compos. Math. 140 (2004), no. 3, 541-551. MRMR2041767 (2004k:11058)

[Koi04] Masao Koike, Thompson series and Ramanujan's identities, Galois theory and modular forms, Dev. Math., vol. 11, Kluwer Acad. Publ., Boston, MA, 2004, pp. 367-373. MRMR2059774 (2005c:11051)

[Mar96] Yves Martin, Multiplicative $\eta$-quotients, Trans. Amer. Math. Soc. 348 (1996), no. 12, 4825-4856. MRMR1376550 (97d:11070)

[Ono04] Ken Ono, The web of modularity: arithmetic of the coefficients of modular forms and $q$-series, CBMS Regional Conference Series in Mathematics, vol. 102, Published for the Conference Board of the Mathematical Sciences, Washington, DC, 2004. MRMR2020489 (2005c:11053)

[Rog21] L. J. Rogers, On a type of modular relations, Proc. London Math. Soc (2) 19 (1921), 387-397.

[Wat33] G. N. Watson, Proof of certain identities in combinatory analysis, J. Indian Math. Soc. 20 (1933), 57-69.

Department of Mathematics, University of Wisconsin, Madison, Wisconsin 53706

E-mail address: bringman@math.wisc.edu

Department of Mathematics, The Ohio State University, Columbus, Ohio 43210

E-mail address: swisher@math.ohio-state.edu 